\title{
Modulation of Dendritic Patterns During Electrodeposition: A Nonlinear Phase-field Model
}

\author{
Lei Chen ${ }^{1,2, ~ *, ~ H a o ~ W e i ~ Z h a n g ~}{ }^{1}$, Lin Yun Liang ${ }^{1}$, Zhe Liu ${ }^{1}$, Yue Qi $^{3}$, Peng Lu ${ }^{4}$, James Chen ${ }^{5}$ \\ and Long-Qing Chen ${ }^{1}$ \\ ${ }^{1}$ Department of Materials Science and Engineering, The Pennsylvania State University, University Park, PA \\ 16802, USA \\ ${ }^{2}$ Department of Mechanical Engineering, Mississippi State University, Mississippi State, MS, 39762 \\ ${ }^{3}$ Department of Chemical Engineering and Materials Science, Michigan State University, East Lansing, \\ Michigan 48824, USA \\ ${ }^{4}$ General Motors Research and Development center, Warren, Michigan 48090, USA \\ ${ }^{5}$ Division of Engineering, Pennsylvania State University, the Altoona College, Altoona, PA 16601, USA
}

\begin{abstract}
A nonlinear phase-field model, accounting for the Butler-Volmer electrochemical reaction kinetics, is developed to investigate the dendritic patterns during an electrodeposition process. Using lithium electrodeposition as an example, the proposed model is first verified by comparison with the Nernst equation in a 1D equilibrium system. The nonlinear electrochemical kinetics is also confirmed at non-equilibrium condition. The dendritic patterns are examined as a function of applied voltage and initial electrode surface morphology. A design map is proposed to tailor the electrode surface morphology and the applied voltage to avoid undesired dendritic patterns.
\end{abstract}

Keywords: Phase-field model; Dendrite; Electrodeposition; Lithium-ion battery

${ }^{*}$ Corresponding author. Phone number: +1-814-777-6248; Fax Number: +1-814-865-7173;

E-mail Address: 1uc28@psu.edu (Lei Chen) 


\section{Introduction}

Electrodeposition has been widely observed in numbers of applications such as electroplating, electroforming, electrocorrosion and battery charging. However, dendrites characterized as multilevel branching usually occur at the electrode-electrolyte interface during electrodeposition processes if they are not carefully controlled [1-4]. Such dendrites generated far from equilibrium have also fascinated scientists for decades due to their important effects on physical and chemical properties of the electrodeposition systems and the performance of electrochemical devices. For example, Lithium (Li) electrodeposition on a Li-metal electrode often takes place in high capacity $\mathrm{Li}_{2} \mathrm{O}_{2}$ (lithium-oxygen) and Li-S (lithium-sulfur) batteries [5-7]. These newly developed high capacity lithium batteries, however, still suffer from unexpectedly failure by short-circuiting via the dendrites that grow even across electrodes upon recharging [8].

The important role of dendrites in electrodeposition systems has stimulated numerous efforts on modulating the dendritic patterns. These works were mostly based on the modification of electrode materials [9], electrode surface morphology [10, 11], solvent and electrolyte composition $[12,13]$ and operational current density or voltage $[14,15]$. The basic idea behind these treatments is to control the kinetics and the instability of interface that are intricately combined.

The present paper aims to formulate a thermodynamically consistent model to predict the dendritic patterns during an electrochemical process using Li-electrodepostion as an example. The first attempt to model the electrochemical dendrite growth was made by Monroe and Newman [16]. They presented a comprehensive mathematical model for 
temporal evolution of dendrite tip height and growth velocity in Li-polymer cells. Recently, Akolkar $[17,18]$ extended this model by incorporating a concentration-dependent diffusion coefficient, with application to liquid electrolytes. More recently, Aryanfar et al. [19] proposed a coarse-grained Monte Carlo calculation to uncover the Li-dendrite mechanism, by dealing explicitly with $\mathrm{Li}^{+}$migration in time-dependent non-uniform electric fields. However, they did not explicitly simulate the temporal evolution of electrode-electrolyte interface.

Phase-field method has been applied to a vast range of phenomena in materials processes, e.g., solidification, solid-state phase transformation, recrystallization, and grain growth $[20,21]$. Phase-field method is formulated based on the theory of irreversible thermodynamics, and is advantageous in addressing the time-dependent evolving morphologies process, which is hard to implement in traditional sharp-interface model [16]. The early attempt along this line was made by Guyer et al. $[22,23]$ who developed a 1-D phase-field model to investigate the equilibrium state and kinetic behavior of electrochemistry. Later, Okajima et al. [24] simulated the 2-D electrodeposition process by linking a Cahn-Hilliard equation with a Butler-Volmer type equation. Recently, Liang et al. [25] proposed a 1-D formulation that captures the Butler-Volmer kinetics of electrodeposition. More recently Ely et al. [26] conducted a phase-field study on the kinetics of $\mathrm{Li}$ electrodeposits by extending the asymptotic analysis of the phase field theory. However, all these models either are assuming a linear electrochemical reaction kinetics that breaks down when the system is highly out of equilibrium [25, 27], e.g., under high charging voltage, or do not capture an apparent dendritic growth of electrodeposits, or are not derived within a thermodynamic framework based on the electrochemical potential, thus leading to the loss of thermodynamic consistency.

In this article, we solve these discrepancies by formulating a thermodynamically consistent phase-field model. In the model, the phase-field evolves nonlinearly with the 
variational electrochemical overpotential that is a function of electrostatic potential and ion concentration. Such treatment allows us to capture the Butler-Volmer electrochemical reaction kinetics naturally. The mass and current conservation equations are further formulated to solve the ion transport and the local electrostatic potential variation, respectively. Anisotropic surface energy at the interface, evidenced by first principles calculations [28], is incorporated in the model. The present phase-field model is generally applicable to any non-equilibrium electrodeposition system exhibiting the dendritic growth.

\section{Phase-field model}

Consider a simple and general electrodeposition, $\mathrm{M}^{n+}$ cations in a binary dilute electrolyte $\mathrm{M}^{n+} \mathrm{A}^{n-}$ react with electrons $\mathrm{e}^{-}$at the surface of the electrode, and are reduced to M-atom. This process can be illustrated by $\mathrm{M}^{n+}+n \mathrm{e}^{-} \rightarrow \mathrm{M}$. For simplicity, we assume a dilute electrolyte solution. Further, the electrons are assumed to be always supplied on the surface of the electrode. The shape of protuberant at the electrode surface represents the initial morphology of electrode-electrolyte interface as shown in Fig.1. As detailed in Appendix A, the Gibbs free energy of the system can be expressed by

$$
G=\int_{V}\left[f_{c h}(\vec{c})+f_{\text {grad }}(\nabla \vec{c})+f_{\text {elec }}(\vec{c}, \phi)\right] \mathrm{d} V,
$$

where $\vec{C}=\left\{c, C_{+}, c_{-}\right\}$is the set of concentrations for $\mathrm{M}$-atom, $\mathrm{M}^{n+}$ cation and $\mathrm{A}^{n-}$ anion respectively, $f_{c h}(\vec{c})$ is the Helmholtz free energy density and $f_{\text {grad }}=1 / 2 \nabla \overrightarrow{\tilde{c}} \cdot \kappa \nabla \overrightarrow{\tilde{c}}$ is the gradient energy density associated with surface energy. The surface energy anisotropy, i.e., its dependence on the orientation of the electrode-electrolyte interface, is introduced in the system by expanding the gradient coefficient as $\kappa(\theta)=\kappa_{0}[1+\delta \cos (\omega \theta)]$, where $\delta$ and $\omega$ are the strength and mode of the anisotropy, $\kappa_{0}$ is related to the surface energy $\gamma, \theta$ is the angle between the normal vector of interface and the reference axis. $\overrightarrow{\tilde{C}}$ is the set of 
dimensionless concentrations as $\left\{\tilde{C}=c / c_{s}, \tilde{C}_{+}=c_{+} / c_{0}, \tilde{C}_{-}=c_{-} / c_{0}\right\}$, where $c_{s}$ is the site density of M-metal and $c_{0}$ the standard bulk concentration of electrolyte solution. $f_{\text {elec }}=\rho_{e} \phi$ is the electrostatic energy density where $\phi$ is the electrostatic potential, and $\rho_{e}$ is the charge density that is expressed as $\rho_{e}=F \sum_{i} z_{i} c_{i}$ where $z_{i}$ is the valence of species $i$ and $F$ is Faraday's constant. A continuous phase-field variable, $\xi$, with a physical correspondence to the dimensionless concentration of $\mathrm{M}$-atom, as $\xi=\tilde{c}$, is introduced to separate the metal and the electrolyte solution during the interface migration. The value of $\xi$ varies continuously from 1 to 0 in the interfacial region, i.e., corresponding to a diffuse-interface description with a finite thickness. The free energy density is then given by

$$
f_{c h}(\vec{c})=g(\tilde{c})+f_{\text {ion }}\left(\tilde{c}_{+}, \tilde{c}_{-}\right)+\sum_{i} c_{i} \mu_{i}^{\Theta}
$$

where $g(\tilde{c})=W \tilde{c}^{2}(1-\tilde{c})^{2}=W \xi^{2}(1-\xi)^{2}$ is an arbitrary double well function to describe the two equilibrium states for the electrode $(\tilde{c}=1)$ and the electrolyte $(\tilde{c}=0)$ respectively. $W / 16$ represents the barrier height. For a dilute electrolyte, $f_{\text {ion }}=c_{0} R T\left(\tilde{c}_{+} \ln \tilde{c}_{+}+\tilde{c}_{-} \ln \tilde{c}_{-}\right)$, without phase separation. The last term in Eq.(2) stands for the free energy density at the standard (reference) state, with $\mu_{i}^{\Theta}$ defined as the reference chemical potential of species $i$, which could be $\mathrm{M}^{n+}$ cations, or electrons $\mathrm{e}^{-}$, or $\mathrm{M}$-atom.

Having these definitions, the electrochemical reaction rate, $R_{e}$, takes the variational form of

$$
R_{e}=-k_{0} \tilde{c}_{+}^{1-\alpha} a_{M}^{\alpha}\left\{\exp \left[\frac{(1-\alpha) n F \eta}{R T}\right]-\exp \left[\frac{-\alpha n F \eta}{R T}\right]\right\}
$$

where $k_{0}$ is the reaction rate constant. The anodic and cathodic charge-transfer coefficients $\alpha_{a}$ and $\alpha_{c}$ satisfy $\alpha_{a}=1-\alpha$ and $\alpha_{c}=\alpha$ with asymmetry factor $0<\alpha<1$. In addition, the overpotential, $\eta$, is defined as 


$$
\eta=\Delta \phi-\Delta \phi^{e q}=\frac{\Delta \mu}{n F}=\frac{1}{n F} \sum_{i} \frac{\delta G}{\delta c_{i}}
$$

This total overpotential is further defined as the sum of the activation overpotential $\eta_{a}$ and the concentration overpotential $\eta_{c}$ (see Appendix $C$ for more details). In Eq.(3), the activity for M-atom, $a_{M}$, is expressed by

$$
C_{s} R T \ln a_{M}=g^{\prime}(\tilde{c})-\kappa \nabla^{2} \tilde{c}=g^{\prime}(\xi)-\kappa \nabla^{2} \xi
$$

based on the definition of activity [27], e.g., for species $i$, which is given by

$$
a_{i}=\exp \left(\frac{1}{R T} \frac{\partial f_{m i x}}{\partial c_{i}}\right)
$$

where $f_{\text {mix }}=f_{c h}+f_{\text {grad }}-\sum_{i} c_{i} \mu_{i}^{\Theta}$ is the mixing free energy density relative to the standard state. The details on the derivation of Eq.(3) is referred to Appendix B.

In the present model, we consider the phase-field evolves by the electrochemical reaction, $R_{e}$, thus

$$
\frac{\partial \xi}{\partial t}=-k_{0} \tilde{c}_{+}^{1-\alpha} a_{M}^{\alpha}\left\{\exp \left[\frac{(1-\alpha) n F \eta}{R T}\right]-\exp \left[\frac{-\alpha n F \eta}{R T}\right]\right\}
$$

Next, let's analyze the driving force deeply in the electrodeposition system, which is contributed by two parts: interfacial free energy and the electrode reaction affinity. The interfacial energy related to the thermal energy $(k T=0.0257 \mathrm{eV})$ is usually small relative to the electrode reaction affinity when a certain large electrostatic potential (e.g., > $0.5 \mathrm{~V}$ ) is applied to the real electrodeposition systems. Therefore, as detailed in Appendix C, the temporal evolution of phase-field is considered linearly proportional to the interfacial free energy and exponentially to the thermodynamics driving force related electrode reaction, that is 


$$
\begin{aligned}
& \frac{\partial \xi}{\partial t}=-L_{\sigma}\left(g^{\prime}(\xi)-\kappa \nabla^{2} \xi\right) \\
& -L_{\eta} h^{\prime}(\xi)\left\{\exp \left[\frac{(1-\alpha) n F \eta_{a}}{R T}\right]-\tilde{c}_{+} \exp \left[\frac{-\alpha n F \eta_{a}}{R T}\right]\right\}
\end{aligned}
$$

where $h(\xi)=\xi^{3}\left(6 \xi^{2}-15 \xi+10\right)$ is an interpolating function, $\eta_{a}=\Delta \phi-E^{\Theta}$ is the activation overpotential, and $E^{\Theta}$ is the standard half-cell potential. $L_{\sigma}$ and $L_{\eta}$ are, respectively, the interface mobility and the reaction-related constant. All of these variables are defined in Appendix C.

For the species diffusion in the electrodepostion system, M-atom is regarded as immobile without diffusion process, while the electrochemical reaction provides a source term for the evolution of $\mathrm{M}^{n+}$ cation. Ignoring the effect of $\mathrm{A}^{n-}$ anion transport, it can be described by

$$
\frac{\partial \tilde{c}_{+}}{\partial t}=\nabla \cdot\left[D^{e f f} \nabla \tilde{c}_{+}+\frac{D^{e f f} \tilde{c}_{+}}{R T} n F \nabla \phi\right]-\frac{c_{s}}{c_{0}} \frac{\partial \xi}{\partial t},
$$

where the effective diffusion coefficient is interpolated by $D^{\text {eff }}=D^{e} h(\xi)+D^{s}(1-h(\xi))$, where $D^{e}$ and $D^{s}$ are the $\mathrm{M}^{n+}$ diffusion coefficients in the electrode (which is almost zero) and the electrolyte solution respectively. The first two terms duplicate the classical NernstPlanck equation. The last term is to describe the accumulation/consuming of $\mathrm{M}^{n+}$ cation due to the electrochemical reaction on the electrode surface. It is related to the electrochemical reaction rate $R_{e}$, which is detailed in Appendix D.

For the electrostatic potential distribution, assuming the charge neutrality in the system, we consider the current density is conserved described by Poisson equation including a source term to represent the charge that enters or leaves due to the electrochemical reaction, as 


$$
\nabla \cdot\left[\sigma^{e f f} \nabla(\phi(r, t))\right]=I_{R}
$$

where the effective conductivity depends on the phase parameter $\sigma^{\text {eff }}=\sigma^{e} h(\xi)+\sigma^{s}(1-h(\xi)), \sigma^{e}$ and $\sigma^{s}$ are the conductivities of electrode and electrolyte solution, respectively, This source term $I_{R}$ is again related to the reaction rate, $R_{e}$, described in Eq.(3) having a form of $I_{R}=n F c_{S} \partial \xi / \partial t$. Note that non-zero $I_{R}$ is only produced at the electrode-electrolyte interface when the system deviates from its equilibrium state.

\section{Numerical results}

We apply the present nonlinear phase-field model to a realistic, $\mathrm{Li}^{+}+\mathrm{e}^{-} \rightarrow \mathrm{Li}$, electrodeposition system, where the electrode is composed of pure Li-metal, while the electrolyte solution includes cation $\left(\mathrm{Li}^{+}\right)$and anion $\left(\mathrm{PF}_{6}^{-}\right)$species. Such reaction is a typical electrode reaction in half cell of Li-ion batteries and the corresponding parameters characterized from experimental studies or modelling references $[16,29,30]$ are detailed in Table 1. The phase-field model is simulated using a finite element method on the platform of COMSOL Multiphysics 4.4, under an adaptive grid. Only the protuberant is initially considered for the electrode in the model to reduce the computational cost, with the size of electrolyte solution set to $500 \times 500 \mu \mathrm{m}$. On the mesh sensitive study, the system mesh size is set as $140 \times 140$ with a minimum grid spacing of $d_{\min }=2 \mu \mathrm{m}$. We use an implicit time integration, with a time step of $\Delta t=0.2 \mathrm{~s}$. The $\mathrm{Li}^{+}$bulk concentration is employed as $c_{0}=1.0 \times 10^{3} \mathrm{~mol} / \mathrm{m}^{3}$. The site density of Li-metal is inverse of the volume of one mole Liatom that is given by $c_{s}=1 / V=\rho_{\mathrm{Li}} / m_{\mathrm{Li}}=7.64 \times 10^{4} \mathrm{~mol} / \mathrm{m}^{3}$, where $m_{\mathrm{Li}}$ and $\rho_{\mathrm{Li}}$ are molar mass and density of Li, respectively. All the parameters are normalized a characteristic energy density $E_{0}=1.5 \times 10^{6} \mathrm{~J} / \mathrm{m}^{3}$, a characteristic length $l_{0}=100 \mu \mathrm{m}$, and a characteristic 
time step $\Delta t_{0}=4000 \mathrm{~s}$. The symmetric factor $\alpha=0.5$ is speculated in this work [31], unless otherwise specified.

\subsection{One-dimensional phase-field model}

First, we investigate the equilibrium electrode-electrolyte potential difference, i.e., the activation overpotential at the electrode-electrolyte interface in a $1 \mathrm{D}$ system without an applied voltage, for the purpose of validation of the proposed phase-field model. An adiabatic boundary condition is employed to calculate three governing equations by fixing the fluxes of Li-atom dendity, $\mathrm{Li}^{+}$concentration and potential at the boundaries to zero. The equilibrium activation overpotential at the electrode-electrolyte interface is examined for various $\mathrm{Li}^{+}$ concentrations of the electrolyte solution: $\tilde{c}_{+}=0.001,0.01,0.1,0.5,1.0,2.0,5.0,10.0$. Fig.2 shows the calculated activation overpotential, $\eta_{a}=\Delta \phi-E^{\Theta}$, as a function of the logarithmic concentration at equilibrium. The standard half-cell potential $E^{\Theta}$ is set as 0 , which is similar to set $\mathrm{Li} / \mathrm{Li}+$ equilibrium potential as zero for Li-ion battery applications. The activation overpotential decreases with decreasing $\mathrm{Li}^{+}$concentration. The gradient of the fitted line is 0.058 that satisfies perfectly the analytical Nernst equation $\eta_{a}=2.303 R T / F \log _{10}\left(\tilde{c}_{+} / a_{M}\right)$, where the activity $a_{M}$ for Li-atom at equilibrium is 1 .

Further, the Butler-Volmer relation between the rate of electrode reaction and the electrochemical overpotential is examined using the 1D system with an applied voltage. Dirichlet boundary condition is used to solve the $\mathrm{Li}^{+}$diffusion and Poisson equations. In order to remove the effect of $\mathrm{Li}^{+}$concentration on such Butler-Volmer kinetics, we set the $\mathrm{Li}^{+}$ concentration in the electrolyte solution as $\tilde{c}_{+}=1.0$. The electrode-electrolyte potential difference is set to $\Delta \phi=-0.2-0 \mathrm{~V}$, giving rise to a range of overpotential values as $\eta=-0.2-0 \mathrm{~V}$. Cases with the reaction rate constant, $L_{\eta}$, equal to $1.0 / \mathrm{s}$ are calculated. The growth velocity of the electrode-electrolyte interface is examined for various overpotential 
values when $\alpha=1.0$ and 0.5 , and the results are plotted in Fig.3. For the purpose of comparison, the analytical solution derived for the sharp-interface limit of phase-field model $[25,32]$

$$
v=-\frac{\kappa L_{\eta}}{\gamma}\left\{\exp \left[\frac{(1-\alpha) n F \eta}{R T}\right]-\exp \left[\frac{-\alpha n F \eta}{R T}\right]\right\}
$$

is also drawn as a solid line in Fig.3, where $\gamma$ is the interfacial energy per unit area. The numerical results obtained using the phase-field model agree well with the analytical solution for both values of $\alpha$. The linear relationship between the interface velocity $v$ and the overpotential $\eta$ is found to satisfied when $\eta$ is small, whereas at larger $\eta$ values, the interface velocity $v$ appears exponentially dependent on $\eta$.

\subsection{Two-dimensional phase-field model}

In order to model the electrochemical dendrite growth, the addition of anisotropy is implemented in the 2D system. The strength of interfacial anisotropy is set as 0.05 (consistent with the slight anisotropic surface energy in $\mathrm{Li}$ metal)[28], and the $\mathrm{Li}^{+}$concentration of the electrolyte solution as $\tilde{c}=1.0$. As before, Dirichlet boundary condition is applied to solve the $\mathrm{Li}^{+}$diffusion and electrostatic Poisson equations.

\subsubsection{The distributions of fields}

We start with a case where an artificial nucleation occurs at the center of electrodeelectrolyte interface to illustrate the distributions of different fields. Fig.4 shows the snapshots of the phase-field order parameter, $\mathrm{Li}^{+}$concentration and electric potential distributions as a function of evolution time by solving Eqs.(8-10). The local variations of $\mathrm{Li}^{+}$concentration and electric potential are both clearly seen. During the charging operation, the growth of phase-field (i.e., electrodeposit) can be related to the $\mathrm{Li}^{+}$concentration and electric potential by reaction kinetics in which surface tension (i.e., interfacial energy) also appears. This in turn results in a concentration gradient as well in an electric potential 
gradient at the neighborhood of the electrode and/or the deposit. The tips of deposits have larger concentration and electric potential gradients which give larger overpotential and force their faster growth. We should note that the overpotential, as a function of $\mathrm{Li}^{+}$concentration and electric potential, is taken as a field in our simulation which is different from the mathematical model that always taken as a single value. The overpotential across the interface is automatically taken as the thermodynamic driving force based on Eq.(8). The lithium deposition begins when the overpotential is less than zero.

\subsubsection{Dendritic patterns}

In order to statistically characterize the dendritic pattern, we simulate the dendrite growth with a range of applied voltages and protuberant morphologies, which are quantified by the ratio of $b / a$ shown in Fig.1. The distance between each protuberant is $80 \mu \mathrm{m}$. For all the cases examined, Fig.5(a) shows a typical fiber-like pattern with small branches under a small applied voltage of $-0.45 \mathrm{~V}$ and a relatively large $b / a$ value of 8.0 , which agrees with the experiemental observations $[4,11,33,34]$. The fibers grow parallel to the direction of the applied electric field. Lowering the value of $b / a$ and increasing the applied voltage give rise to the pattern of dendrites with side branches, for which the truck follows along the the direction of the applied electric field too. Fig.5(b) shows a typical dendritic pattern with a applied voltage of $-1.5 \mathrm{~V}$ and a $b / a$ value of 4.0, similar to the experimental data in [35]. Further lowering $b / a$ to 2.0 and increasing the applied voltage to $-3.0 \mathrm{~V}$, corresponding to a large driving force, even promotes the tendency of tip to split (see Fig.5(c)) that is in agreement with the finding in experimental reports in $[4,36]$. For the comparison purpose, we also plot the in situ experimentally observed results in Fig.5(d) using the apparatus described [37], in which charging and discharging were carried out in an optical half-cell. A brushed piece of Li foil acted as the negative electrode, while a porous graphite electrode cut from an LR1865AH 18650* laptop battery made by Tianjin Lishen Battery Co. served as the positive 
electrode. The electrode material coated both sides of a copper current collector. Similar to the phase-field model, a $1 \mathrm{M}$ solution of $\mathrm{LiPF}_{6}$ was deposited on the Li electrode. Cells were placed under either current or voltage control using a high precision source/measure unit (Keithley 237) in an external circuit. At an current density of $5 \mathrm{~mA} / \mathrm{cm}^{2}$, two dendrite branches grow into a mossy region on the lower right corner in Fig.5(d), which is apparently consistent with the simulated tip-splitting dendritic pattern in Fig.5(c).

To unravel the underlying mechanism of the transition from the fiber-like, fully dendritic to tip-splitting dendritic patterns, we track the temporal evolution of the average dendritic tip radius for these representative cases, and plot the results in Fig.6. We found that the tip radius of fiber-like pattern begins to decrease rapidly after charging, and then is kept at a small constant value. For the fully dendritic pattern, the tip radius still decreases at the initial state in despite of a relatively small amplitude, thereafter, stays at an almost constant value with a fraction of oscillation. For the third case, the tip radius exhibits an obvious oscillatory evolution which is attributed to the unstable tip splitting. In principle, the smaller tip radius, the larger concentration and overpotential gradients in the neighborhood which give larger electrodeposition rate and force their faster growth. Therefore, the front of Lideposit grows much faster than the behind once its initiallisation for the fibre-like case. As the tip radius increases, the distributions of concentration and overpotential become relatively homogenous, the side branthes start to emerge at the points where sufficient driving force can be achieved if some noises are somehow provided. Once the drving force (e.g., with high applied voltage) is large enough, the tip radius tends to increase more, and the tip appears to split in a random manner which in turn decreases the rip radius. These interaction contributes to the vibration of tip radius during the electrodeposition.

\subsubsection{Design map}

Next, we characterize the transition discussed above, by noting that the length $\lambda$ of 
branching array tilted at an angle to the truck ( $x$-axis) must satisfy the selection criterion $\lambda>\chi l_{T}$, where $\chi$ is the proportionality constant. Here, we set $\chi=0.03$ as reference [38] did. Using this selection criterion on more simulation cases with different applied voltages and protuberant morphologies, we define a phase-like diagram to identity the dendritic patterns with $x$-axis of applied voltage, as well as $y$-axis of the size ratio of protuberant $b / a$ as shown in Fig.6. Examination of the $\Delta \phi(b / a)$ phase diagram also predicts that there will be a transition from the fiber-like to tip-splitting dendritic patterns, as the applied voltage is increased or the protuberant is relatively flat with a small $b / a$ value. We also note that at sufficiently large applied voltage, the unstable tip-splitting always appears regardless of the morphology of protuberant at the electrode-electrolyte interface.

\section{Conclusions}

In summary, a thermodynamically consistent phase-field model, accounting for the nonlinear reaction kinetics, has been proposed to investigate the dendritic patterns during an electrodeposition process. The model has been validated by comparing the equilibrium electrode-electrolyte potential difference with the Nernst equation, taking an example of Lielectrodeposition on Li-metal. Then we have reproduced the Butler-Volmer nonlinear electrochemical kinetics in a $1 \mathrm{D}$ non-equilibrium system. Three different dendritic patterns have been discovered depending on the applied voltage and the interface morphology. A phase diagram was proposed, which could potentially be used as the guidance to experimentally control of Li-dendrite patterns. Analysis on the dendritic patterns demonstrates that the large applied voltage or the flat protuberant at the interface contributes to the side branches of dendrites, and even promotes an unstable tip-splitting.

Finally, we would like to emphasize that electrodeposition is a versatile technique but having a complex process. Controlling the dendrites that occur at the electrode-electrolyte 
interface relies on a number of external or internal factors. In the present article, we focus on the effects of applied voltage and initial electrode morphology on the dendritic patterns during charging. Besides such two factors, the deposit patterns could also be attributed to, such as interfacial properties [10, 11], electrode material properties [9], electrolyte solution composition and properties $[12,13]$, evolution time [39, 40], applied voltage type (e.g., pulse) $[9,13,15,16]$, etc. The solid-electrolyte-interface (SEI) layer [41, 42], recognized acting as significant role during electrodeposition, has also not been explicitly considered. The inclusion of such SEI layer in the phase-field model is under way, in order to investigate the role of SEI layer in the locations of dendritic nucleation as well as the morphologies of dendritic growth. A robust model that is capable of simulating the dendrite growth during electrodeposition, is not only interesting from the fundamental aspect, but also important for the design of electrochemical systems in practice. The present nonlinear phase-fiend model established herein gives us a new pathway for the further study of these issues.

\section{Acknowledgments}

The authors are grateful for the financial support by NSF under CMMI-1235092. The computer simulations were carried out on the LION clusters at the Pennsylvania State University. 


\section{Appendix A: Gibbs free energy functional}

Here we provide the detailed procedure to obtain the expression for Gibbs free energy functional of the electrodeposition system

$$
G=\int_{V} f(\vec{c}, \phi) \mathrm{d} V
$$

where $f(\vec{c}, \phi)$ is the Gibbs free energy density. $\phi$ is the electrostatic potential and $\vec{c}=\left\{c, c_{+}, c_{-}\right\}$is the set of concentrations for $\mathrm{M}$-atom, $\mathrm{M}^{n+}$ cation and $\mathrm{A}^{n_{-}}$anion respectively. Further, $\overrightarrow{\tilde{c}}$ is defined as the set of dimensionless concentrations as $\left\{\tilde{c}=c / c_{s}, \tilde{c}_{+}=c_{+} / c_{0}, \tilde{C}_{-}=c_{-} / c_{0}\right\}$, where $c_{s}$ is the site density of M-metal and $c_{0}$ the bulk concentration of electrolyte solution.

It is convenient to identify the classical chemical potential

$$
\mu_{i}=R T \ln a_{i}+\mu_{i}^{\Theta}=R T \ln \tilde{c}_{i}+\mu_{i}^{e x},
$$

and the classical electrochemical potential

$$
\bar{\mu}_{i}=R T \ln a_{i}+\mu_{i}^{\Theta}+F z_{i} \phi=R T \ln \tilde{c}_{i}+\bar{\mu}_{i}^{e x},
$$

where $R T$ is the product of the molar gas constant, $R$, and the temperature, T. $F$ is the Faraday's constant. $z_{i}$ and $a_{i}$ are the valence and the activity of component $i$ respectively. $\mu_{i}^{\Theta}$ denotes the reference chemical potential of species $i . \mu_{i}^{e x}$ and $\bar{\mu}_{i}^{e x}$ are respectively the excess chemical potential and the excess electrochemical potential. The activity coefficient $\gamma_{i}$ expressed by

$$
\gamma_{i}=\exp \left[\left(\mu_{i}^{e x}-\mu_{i}^{\Theta}\right) / R T\right]
$$

or

$$
\gamma_{i}=\exp \left[\left(\bar{\mu}_{i}^{e x}-\mu_{i}^{\Theta}-F Z_{i} \phi\right) / R T\right]
$$

is a measure of non-ideality $\left(a_{i}=\gamma_{i} \tilde{c}_{i}\right)$ 
Assuming the M-metal and electrolyte solution are added into the system without any interaction, it is convenient to introduce the electrochemical free energy density in a homogenous system

$$
f_{e l+c h}=\sum_{i} c_{i} \bar{\mu}_{i}=\sum_{i} c_{i}\left(R T \ln a_{i}+\mu_{i}^{\Theta}+F z_{i} \phi\right) .
$$

Further considering a dilute electrolyte solution with $a_{i}=\tilde{c}_{i}$, it can be written as

$$
\begin{aligned}
f_{e l+c h} & =R T \sum_{i} c_{i} \ln \tilde{c}_{i}+\sum_{i} c_{i} \mu_{i}^{\Theta}+\sum_{i} F z_{i} c_{i} \phi \\
& =R T \sum_{i} c_{i} \ln \tilde{c}_{i}+\sum_{i} c_{i} \mu_{i}^{\Theta}+\rho_{e} \phi \\
& =c_{s} R T \tilde{c} \ln \tilde{c}+c_{0} R T\left(\tilde{c}_{+} \ln \tilde{c}_{+}+\tilde{c}_{-} \ln \tilde{c}_{-}\right)+\sum_{i} c_{i} \mu_{i}^{\Theta}+\rho_{e} \phi
\end{aligned}
$$

where the term $c_{s} R T \tilde{c} \ln \tilde{c}$ corresponds to the contribution of M-atom, of which the dimensionless concentration $\tilde{c}=1$ in the M-metal and $\tilde{c}=0$ in the electrolyte solution. We accordingly have $C_{S} R T \tilde{c} \ln \tilde{c}=0$ if the sharp interface between the M-metal and the electrolyte solution is assumed.

In order to describe the diffuse interface in the present phase-field model, a continuous phase-field variable, $\xi$, with a physical correspondence to the dimensionless concentration of M-atom, as $\xi=\tilde{c}$, is introduced. An arbitrary double well function $g(\tilde{c})=W \tilde{c}^{2}(1-\tilde{c})^{2}=W \xi^{2}(1-\xi)^{2}$ is used to describe the two equilibrium states for the electrode $(\tilde{c}=1)$ and the electrolyte $(\tilde{c}=0)$. W/16 represents the barrier height. Thus, the electrochemical free energy density reads

$$
f_{e l+c h}=W \tilde{c}^{2}(1-\tilde{c})^{2}+c_{0} R T\left(\tilde{c}_{+} \ln \tilde{c}_{+}+\tilde{c}_{-} \ln \tilde{c}_{-}\right)+\sum_{i} c_{i} \mu_{i}^{\Theta}+\rho_{e} \phi,
$$

or

$$
f_{e l+c h}=W \xi^{2}(1-\xi)^{2}+c_{0} R T\left(\tilde{c}_{+} \ln \tilde{c}_{+}+\tilde{c}_{-} \ln \tilde{c}_{-}\right)+\sum_{i} c_{i} \mu_{i}^{\Theta}+\rho_{e} \phi
$$

In addition, the free energy density associated with the gradient is routinely given by 


$$
f_{\text {grad }}=1 / 2 \nabla \overrightarrow{\tilde{C}} \cdot \kappa \nabla \overrightarrow{\tilde{C}}
$$

where $\kappa$ is the gradient coefficient.

Combing Eqs.(A6-A8) yields the Gibbs free energy density

$$
\begin{aligned}
& f(\vec{c}, \phi)=f_{\text {el+ch }}+f_{\text {grad }} \\
& =W \tilde{c}^{2}(1-\tilde{c})^{2}+c_{0} R T\left(\tilde{c}_{+} \ln \tilde{c}_{+}+\tilde{c}_{-} \ln \tilde{c}_{-}\right)+\sum_{i} c_{i} \mu_{i}^{\Theta}+\rho_{e} \phi+1 / 2 \nabla \overrightarrow{\tilde{c}} \cdot \kappa \nabla \overrightarrow{\tilde{c}}
\end{aligned}
$$

Therefore, we have

$$
\begin{aligned}
& f_{c h}(\vec{c})=W \tilde{c}^{2}(1-\tilde{c})^{2}+c_{0} R T\left(\tilde{c}_{+} \ln \tilde{c}_{+}+\tilde{c}_{-} \ln \tilde{c}_{-}\right)+\sum_{i} c_{i} \mu_{i}^{\Theta} \\
& f_{\text {grad }}(\nabla \vec{c})=1 / 2 \nabla \overrightarrow{\tilde{c}} \cdot \kappa \nabla \overrightarrow{\tilde{c}} \\
& f_{\text {elec }}=\rho_{e} \phi=F \sum_{i} z_{i} c_{i} \phi
\end{aligned}
$$




\section{Appendix B: Electrochemical reaction kinetics}

In a consistent formulation of electrochemical reaction kinetics [27, 43], the reaction $\left(S_{1} \rightarrow S_{2}\right)$ rate, $R_{e}$, is expressed by the excess electrochemical potential at the different states, as

$$
R_{e}=-k_{0} \tilde{c}_{1} \exp \left[\frac{-\left(\bar{\mu}_{t}^{e x}-\bar{\mu}_{1}^{e x}\right)}{R T}\right]+k_{0} \tilde{c}_{2} \exp \left[\frac{-\left(\bar{\mu}_{t}^{e x}-\bar{\mu}_{2}^{e x}\right)}{R T}\right],
$$

where $k_{0}$ is the reaction constant. Local minima of $\bar{\mu}_{1}^{e x}$ and $\bar{\mu}_{2}^{e x}$ corresponds to the excess electrochemical potential at state 1 and state 2 respectively, with transition over an activation barrier $\bar{\mu}_{t}^{e x}$ as shown in Fig.A1. Enforcing detailed balance $\left(R_{e}=0\right)$ in equilibrium i.e., the electrochemical potential given in Eq.(A15) equals at two states, $\bar{\mu}_{1}=\bar{\mu}_{2}$, yields the reaction rate that is consistent with the following non-equilibrium expression

$$
R_{e}=-k_{0}\left\{\exp \left[\frac{-\left(\bar{\mu}_{t}^{e x}-\bar{\mu}_{1}\right)}{R T}\right]-\exp \left[\frac{-\left(\bar{\mu}_{t}^{e x}-\bar{\mu}_{2}\right)}{R T}\right]\right\} \text {. }
$$

Having the definition of the electrochemical potential, it is convenient to write the expressions for different components in the electrodeposition reaction, $\mathrm{M}^{n+}+n \mathrm{e}^{-} \rightarrow \mathrm{M}$

$$
\begin{aligned}
& \bar{\mu}_{M^{n+}}=R T \ln a_{M^{n+}}+\mu_{M^{n+}}^{\Theta}+n F \phi_{s}, \\
& \bar{\mu}_{e}=R T \ln a_{e}+\mu_{e}^{\Theta}-F \phi_{e}, \\
& \bar{\mu}_{M}=R T \ln a_{M}+\mu_{M}^{\Theta},
\end{aligned}
$$

where $\phi_{s}$ and $\phi_{e}$ are, respectively, the electrostatic potential in the electrolyte solution and the electrode. The electrode-electrolyte interfacial potential difference is $\Delta \phi=\phi_{e}-\phi_{s}$. Applying Eq.(A16) to the present electrodeposition reaction with $\bar{\mu}_{1}=\bar{\mu}_{M^{n+}}+n \bar{\mu}_{e}, \bar{\mu}_{2}=\bar{\mu}_{M}$ and $\Delta \mu=\bar{\mu}_{2}-\bar{\mu}_{1}$, the electrode-electrolyte potential difference at equilibrium with $\Delta \mu=0$, is given by the Nernst equation 


$$
\Delta \phi^{e q}=E^{\Theta}+\frac{R T}{n F} \ln \frac{a_{M^{n+}} a_{e}^{n}}{a_{M}},
$$

where

$$
E^{\Theta}=\frac{\mu_{M^{n+}}^{\Theta}+n \mu_{e}^{\Theta}-\mu_{M}^{\Theta}}{n F}
$$

is the standard half-cell potential or is standard potential difference between reactants and products. Assuming a dilute electrolyte solution and the activity for electron is unity, Eq.(A20) is further written as

$$
\Delta \phi^{e q}=E^{\Theta}+\frac{R T}{n F} \ln \frac{\tilde{c}_{+}}{a_{M}} .
$$

Out of equilibrium, the reaction rate, $R_{e}$, or the current, $I=n e R_{e}$, is controlled by the overpotential, $\eta$, which is defined as [44]

$$
\eta=\Delta \phi-\Delta \phi^{e q}=\Delta \phi-E^{\Theta}-\frac{R T}{n F} \ln \frac{\tilde{c}_{+}}{a_{M}}=\frac{\Delta \mu}{n F}=\frac{1}{n F} \sum_{i} \frac{\delta G}{\delta c_{i}}
$$

Thus, we have

$$
R_{e}=-R_{0}\left\{\exp \left[\frac{(1-\alpha) \Delta \mu}{R T}\right]-\exp \left[\frac{-\alpha \Delta \mu}{R T}\right]\right\}=-R_{0}\left\{\exp \left[\frac{(1-\alpha) n F \eta}{R T}\right]-\exp \left[\frac{-\alpha n F \eta}{R T}\right]\right\}
$$

and

$$
I=-I_{0}\left\{\exp \left[\frac{(1-\alpha) \Delta \mu}{R T}\right]-\exp \left[\frac{-\alpha \Delta \mu}{R T}\right]\right\}=-I_{0}\left\{\exp \left[\frac{(1-\alpha) n F \eta}{R T}\right]-\exp \left[\frac{-\alpha n F \eta}{R T}\right]\right\}
$$

where $R_{0}$ and $I_{0}$ are the exchange reaction rate and current respectively. The anodic and cathodic charge-transfer coefficients $\alpha_{a}$ and $\alpha_{c}$ satisfy $\alpha_{a}=1-\alpha$ and $\alpha_{c}=\alpha$ with asymmetry factor $0<\alpha<1$. If $\eta<0, R_{e}>0$ corresponds to the reduction of the electrolyte; whereas if $\eta>0$, the electrode is being oxidized with $R_{e}<0$, which is easily illustrated by the landscape of the excess electrochemical potential along the reaction coordinate as schematically shown in Fig.A1. 
In order to model the Butler-Volmer kinetics, the excess electrochemical potential for transition state is defined as $[27,44]$

$$
\bar{\mu}_{t}^{e x}=R T \ln \gamma_{t}+(1-\alpha)\left(n F \phi_{s}-n F \phi_{e}+\mu_{M^{n+}}^{\Theta}+n \mu_{e}^{\Theta}\right)+\alpha \mu_{M}^{\Theta},
$$

which yields

$$
R_{0}=k_{0} \tilde{c}_{+}^{1-\alpha} a_{M}^{\alpha} / \gamma_{t} \text { and } I_{0}=n e k_{0} \tilde{c}_{+}^{1-\alpha} a_{M}^{\alpha} / \gamma_{t}
$$

where the activity coefficient at the transition state $\gamma_{t}$ is obtained based on the double well function describing the diffuse interface, i.e., $g(\xi)=W \xi^{2}(1-\xi)^{2}$ as

$$
R T \ln \gamma_{t}=g^{\prime}(\xi)-\kappa \nabla^{2} \xi-R T \ln \xi,
$$




\section{Appendix C: Phase-field equation}

Combining Eqs. (A23-26) leads to the reaction rate

$$
R_{e}=-R_{0}\left\{\exp \left[\frac{(1-\alpha) n F \eta}{R T}\right]-\exp \left[\frac{-\alpha n F \eta}{R T}\right]\right\}
$$

where the total overpotential $\eta=\eta_{a}+\eta_{c}$ is the sum of the activation overpotential and the concentration overpotential. The activation overpotential is defined as

$$
\eta_{a}=\Delta \phi-E^{\Theta}
$$

Subtracting Eq.(A28) from Eq.(A22), the concentration overpotential is expressed by

$$
\eta_{c}=-\frac{R T}{n F} \ln \frac{\tilde{c}_{+}}{a_{M}}=-\frac{R T}{n F}\left(\ln \tilde{c}_{+}-\ln a_{M}\right)
$$

In Eq.(A27), the activity for M-atom, $a_{M}$, is expressed by

$$
c_{S} R T \ln a_{M}=g^{\prime}(\tilde{c})-\kappa \nabla^{2} \tilde{c}=g^{\prime}(\xi)-\kappa \nabla^{2} \xi
$$

based on the definition of activity [27], e.g., for species $i$, which is given by

$$
a_{i}=\exp \left(\frac{1}{R T} \frac{\partial f_{m i x}}{\partial c_{i}}\right)
$$

where $f_{\text {mix }}=f_{c h}+f_{\text {grad }}-\sum_{i} c_{i} \mu_{i}^{\Theta}$ is the mixing free energy density relative to the standard state.

Substituting the concentration overpotential, $\eta_{c}$, into Eq.(A29), we have

$$
R_{e}=-R_{0}\left\{\exp \left[(1-\alpha)\left(\frac{n e \eta_{\alpha}}{R T}-\ln \tilde{c}_{+}+\ln a_{M}\right)\right]-\exp \left[-\alpha\left(\frac{n e \eta_{\alpha}}{R T}-\ln \tilde{c}_{+}+\ln a_{M}\right)\right]\right\}
$$

Considering the electrodeposition system physically, the driving force is generally contributed by two parts: interfacial free energy and the electrode reaction affinity. Thus, we write the reaction rate, $R_{e}$, as the accumulation of these two parts

$$
R_{e}=R_{\sigma}+R_{\eta}
$$


where $R_{\sigma}$ corresponds to the driving force for interfacial energy and $R_{\eta}$ is the driving force for the electrochemcial reaction. Since $a_{M}$ involves the gradient energy density term from Eq.(A30), $R_{\sigma}$ is considered to be expressed by the term $\ln a_{M}$ within parentheses in Eq.(A32). In order to illustrate such separation, we further write Eq.(A32) as

$$
R_{e}=-R_{0}\{\exp [(1-\alpha)(x+y)]-\exp [-\alpha(x+y)]\}
$$

where $x=\frac{n e \eta_{\alpha}}{R T}-\ln \tilde{c}_{+}$corresponding to $R_{\eta}$ and $y=\ln a_{M}$ for $R_{\sigma}$.

When the system is far from equilibrium, $R_{\sigma}$, is usually much smaller than $R_{\eta}$, therefore, $y \square x$. Performing Taylor expansion on Eq.(A34), we have

$$
\begin{aligned}
& R_{e}=-R_{0}\{\exp [(1-\alpha)(x+y)]-\exp [-\alpha(x+y)]\} \\
& =-R_{0}\{\exp [(1-\alpha) x]+(1-\alpha) \exp [(1-\alpha) x] y-\exp (-\alpha x)+\alpha \exp (-\alpha x) y\}, \\
& =-R_{0}\{\exp [(1-\alpha) x]-\exp (-\alpha x)\}-R_{0} y\{(1-\alpha) \exp [(1-\alpha) x]+\alpha \exp (-\alpha x)\}
\end{aligned}
$$

with

$$
R_{\sigma}=-R_{0}\{(1-\alpha) \exp [(1-\alpha) x]+\alpha \exp (-\alpha x)\} y
$$

and

$$
R_{\eta}=-R_{0}\{\exp [(1-\alpha) x]-\exp (-\alpha x)\}
$$

where $R_{\sigma}$ is linearly proportional to $y$ and $R_{\eta}$ is non-linearly proportional to $x$ with a Butler-Volmer relation. Ignoring the dependence of $R_{\sigma}$ on $x$ or the dependence of $R_{\eta}$ on $y$, letting

$$
L_{\sigma}=R_{0}\{(1-\alpha) \exp [(1-\alpha) x]+\alpha \exp (-\alpha x)\} / c_{s} R T
$$

as the interfacial mobility having a constant value, and substituting Eq.(A30) into $R_{\sigma}$, we then have 


$$
R_{\sigma}=-L_{\sigma}\left(g^{\prime}(\xi)-\kappa \nabla^{2} \xi\right)
$$

In addition, substituting $x=\frac{n e \eta_{\alpha}}{R T}-\ln \tilde{c}_{+}$and $R_{0}=k_{0} \tilde{c}_{+}^{1-\alpha} a_{M}^{\alpha} / \gamma_{t}$ into Eq.(A37), $R_{\eta}$ is expressed as

$$
\begin{aligned}
& R_{\eta}=-k_{0} \tilde{c}_{+}^{1-\alpha} a_{M}^{\alpha} / \gamma_{t}\left\{\exp \left[(1-\alpha)\left(\frac{n e \eta_{\alpha}}{R T}-\ln \tilde{c}_{+}\right)\right]-\exp \left[-\alpha\left(\frac{n e \eta_{\alpha}}{R T}-\ln \tilde{c}_{+}\right)\right]\right\} \\
& =-k_{0} a_{M}^{\alpha} / \gamma_{t}\left\{\exp \left[(1-\alpha)\left(\frac{n F \eta_{\alpha}}{R T}\right)\right]-\tilde{c}_{+} \exp \left[-\alpha\left(\frac{n e \eta_{\alpha}}{R T}\right)\right]\right\}
\end{aligned}
$$

In addition, for $R_{\eta}$, Eq.(A40) only represents the driving force from reactants to products in the form of sharp interface limit. In order to describe such electrochemcial reaction at the electrode-electrolyte diffuse interface, an interpolating function $h^{\prime}(\xi)=30 \xi^{2}(1-\xi)^{2}$ is introduced for $R_{\eta}$. Thus, the phase-field evolves by

$$
\begin{aligned}
& \frac{\partial \xi}{\partial t}=-L_{\sigma}\left(g^{\prime}(\xi)-\kappa \nabla^{2} \xi\right) \\
& -L_{\eta} h^{\prime}(\xi)\left\{\exp \left[\frac{(1-\alpha) n F \eta_{a}}{R T}\right]-\tilde{c}_{+} \exp \left[\frac{-\alpha n F \eta_{a}}{R T}\right]\right\}^{\prime}
\end{aligned}
$$

where $L_{\eta}=k_{0} a_{M}^{\alpha} / \gamma_{t}$ that is also regarded as a constant in this work. 


\section{Appendix D: Diffusion equation}

For the species diffusion in the electrodepostion system, the electrochemical reaction provides a source term for the evolution of species which can be described by the set of following equations

$$
\frac{\partial c_{i}}{\partial t}=\nabla \cdot\left[\frac{D_{i} c_{i}}{R T} \nabla \frac{\delta G}{\delta c_{i}}\right] \mp c_{s} R_{e}\left(\frac{n F \eta}{R T}\right),
$$

where signal "-" corresponds to the reactant ( $\mathrm{M}^{n+}$ cation), while signal "+" for the product (M-atom). It is worth noting that Eq. (A44) implies the total amount of $\mathrm{M}^{n+}$ elimination in the electrolyte solution is equivalent to the total amount of $\mathrm{M}$ deposition on the surface of metal electrode. Further, the M-atom is regarded as immobile, accordingly, $D_{i}=0$, thus, the diffusion of M-atom is governed by

$$
\frac{\partial c}{\partial t}=c_{s} R_{e}\left(\frac{n F \eta}{R T}\right) \text { or } \frac{\partial \tilde{c}}{\partial t}=R_{e}\left(\frac{n F \eta}{R T}\right) .
$$

The $\mathrm{M}^{n+}$ cation diffuses following

$$
\frac{\partial c_{+}}{\partial t}=\nabla \cdot\left[D^{e f f} \nabla \mathrm{c}_{+}+\frac{D^{e f f} C_{+}}{R T} n F \nabla \phi\right]-c_{s} R_{e}\left(\frac{n F \eta}{R T}\right)
$$

Combining Eqs. (A43-44) yields

$$
\frac{\partial \tilde{c}_{+}}{\partial t}=\nabla \cdot\left[D^{e f f} \nabla \tilde{c}_{+}+\frac{D^{e f f} \tilde{c}_{+}}{R T} n F \nabla \phi\right]-\frac{c_{s}}{c_{0}} \frac{\partial \tilde{c}}{\partial t} .
$$




\section{References}

[1] J. Barton, J.M. Bockris, Proceedings of the Royal Society of London. Series A. Mathematical and Physical Sciences, 268 (1962) 485-505.

[2] J. Diggle, A. Despic, J.M. Bockris, Journal of The Electrochemical Society, 116 (1969) 1503-1514.

[3] M. Pasquale, D. Barkey, A. Arvia, Journal of The Electrochemical Society, 152 (2005) C149-C157.

[4] M. Sun, H.-G. Liao, K. Niu, H. Zheng, Scientific reports, 3 (2013).

[5] M. Armand, J.-M. Tarascon, Nature, 451 (2008) 652-657.

[6] K.J. Harry, D.T. Hallinan, D.Y. Parkinson, A.A. MacDowell, N.P. Balsara, Nature materials, 13 (2014) 69-73.

[7] B. Huskinson, M.P. Marshak, C. Suh, S. Er, M.R. Gerhardt, C.J. Galvin, X. Chen, A. Aspuru-Guzik, R.G. Gordon, M.J. Aziz, Nature, 505 (2014) 195-198.

[8] J.B. Goodenough, Journal of Solid State Electrochemistry, 16 (2012) 2019-2029.

[9] I.W. Seong, C.H. Hong, B.K. Kim, W.Y. Yoon, Journal of Power Sources, 178 (2008) 769-773.

[10] T. Nishida, K. Nishikawa, M. Rosso, Y. Fukunaka, Electrochimica Acta, 100 (2013) 333-341.

[11] K. Nishikawa, T. Mori, T. Nishida, Y. Fukunaka, M. Rosso, T. Homma, Journal of The Electrochemical Society, 157 (2010) A1212-A1217.

[12] A. Basile, A.F. Hollenkamp, A.I. Bhatt, A.P. O'Mullane, Electrochemistry Communications, 27 (2013) 69-72.

[13] O. Crowther, A.C. West, Journal of The Electrochemical Society, 155 (2008) A806-A811.

[14] C. Brissot, M. Rosso, J.N. Chazalviel, S. Lascaud, Journal of Power Sources, 81-82 (1999) 925-929.

[15] M.Z. Mayers, J.W. Kaminski, T.F. Miller III, The Journal of Physical Chemistry C, 116 (2012) 26214-26221.

[16] C. Monroe, J. Newman, Journal of The Electrochemical Society, 150 (2003) A1377-A1384.

[17] R. Akolkar, Journal of Power Sources, 246 (2014) 84-89.

[18] R. Akolkar, Journal of Power Sources, 232 (2013) 23-28.

[19] A. Aryanfar, D. Brooks, B.V. Merinov, W.A. Goddard, A.J. Colussi, M.R. Hoffmann, The Journal of Physical Chemistry Letters, (2014).

[20] L. Chen, J. Chen, R. Lebensohn, Y. Ji, T. Heo, S. Bhattacharyya, K. Chang, S. Mathaudhu, Z. Liu, L. Chen, Computer Methods in Applied Mechanics and Engineering, (2014).

[21] L.-Q. Chen, Annual review of materials research, 32 (2002) 113-140.

[22] J.E. Guyer, W.J. Boettinger, J.A. Warren, G.B. McFadden, Physical Review E, 69 (2004) 021603.

[23] J.E. Guyer, W.J. Boettinger, J.A. Warren, G.B. McFadden, Physical Review E, 69 (2004) 021604.

[24] Y. Shibuta, Y. Okajima, T. Suzuki, Science and Technology of Advanced Materials, 8 (2007) 511-518.

[25] L. Liang, Y. Qi, F. Xue, S. Bhattacharya, S.J. Harris, L.-Q. Chen, Physical Review E, 86 (2012) 051609.

[26] D.R. Ely, A. Jana, R.E. García, Journal of Power Sources, 272 (2014) 581-594.

[27] M.Z. Bazant, Accounts of chemical research, 46 (2013) 1144-1160.

[28] H.W. Zhang, Z. Liu, L.Y. Liang, L. Chen, Y. Qi, S.J. Harris, J. Chen, L.Q. Chen, Journal of Electrochemical Society, (2015).

[29] M. Ota, S. Izuo, K. Nishikawa, Y. Fukunaka, E. Kusaka, R. Ishii, J.R. Selman, Journal of Electroanalytical Chemistry, 559 (2003) 175-183.

[30] L.O. Valøen, J.N. Reimers, Journal of The Electrochemical Society, 152 (2005) A882-A891.

[31] M.W. Verbrugge, B.J. Koch, Journal of The Electrochemical Society, 141 (1994) 3053-3059.

[32] K. Elder, M. Grant, N. Provatas, J. Kosterlitz, Physical Review E, 64 (2001) 021604.

[33] H. Ghassemi, M. Au, N. Chen, P.A. Heiden, R.S. Yassar, Applied Physics Letters, 99 (2011) 123113.

[34] J.-i. Yamaki, S.-i. Tobishima, K. Hayashi, S. Keiichi, Y. Nemoto, M. Arakawa, Journal of Power Sources, 74 (1998) 219-227.

[35] T.-H. Lin, C.-W. Lin, H.-H. Liu, J.-T. Sheu, W.-H. Hung, Chem. Commun., 47 (2011) 2044-2046.

[36] M.S. Park, S.B. Ma, D.J. Lee, D. Im, S.-G. Doo, O. Yamamoto, Scientific reports, 4 (2014).

[37] S.J. Harris, A. Timmons, D.R. Baker, C. Monroe, Chemical Physics Letters, 485 (2010) 265-274.

[38] N. Provatas, Q. Wang, M. Haataja, M. Grant, Physical Review Letters, 91 (2003) 155502.

[39] M. Rosso, C. Brissot, A. Teyssot, M. Dollé, L. Sannier, J.-M. Tarascon, R. Bouchet, S. Lascaud, Electrochimica Acta, 51 (2006) 5334-5340.

[40] M. Rosso, E. Chassaing, J.N. Chazalviel, T. Gobron, Electrochimica Acta, 47 (2002) 1267-1273.

[41] E. Peled, Journal of Power Sources, 9 (1983) 253-266.

[42] S.S. Zhang, K. Xu, T.R. Jow, Electrochimica Acta, 51 (2006) 1636-1640.

[43] K. Sekimoto, in: Lecture Notes in Physics, Berlin Springer Verlag, 2010.

[44] A.J. Bard, L.R. Faulkner, Electrochemical methods: fundamentals and applications, Wiley New York, 1980. 


\section{List of figures}

Fig.1. Schematic-diagram showing a representative electrodeposition system. A protuberant of size $a \times b$ is present on the electrode surface.

Fig.2. Equilibrium electrode-electrolyte potential difference, i.e., the activation overpotential at the interface as a function of logarithmic concentration in a 1D system.

Fig.3. Absolute value of the growth velocity of interface as a function of the overpotential in the cases of $\alpha=1.0$ and 0.5 . Symbols represent the numerical results and the solid lines represent the analytical solutions from the Butler-Volmer equation. The dotted line is a plot of a linear reaction-overpotential relation for reference.

Fig.4. The snapshots of order parameter $\xi, \mathrm{Li}^{+}$concentration $\tilde{c}$, and electric potential $\phi$ at various evolution times.

Fig.5. Three representative simulated deposits with (a) fiber-like, (b) fully dendritic and (c) tip-splitting dendritic patterns, and (d) the experimentally observed dendritic pattern, in which an optical microscope was utilized to monitor in situ growth of dendrites using the apparatus described [37]. Two dendrite branches grow into a mossy region on the lower right corner, which is apparently consistent with the simulated tip-splitting dendritic pattern in (c).

Fig.6. Temporal variation of the dendritic tip radius for different cases.

Fig.7. Diagram showing the effect of applied voltage and initial protuberant morphology on the transition of electrodeposition patterns. Two fitted boundary lines, red and purple, divide the transition area into three different zones, i.e., fiber-like, fully dendritic and tipsplitting dendritic patterns. The red line is the boundary for transition from fiber-like to fully dendritic patterns, while the purple line is for the transition from fully dendritic to tipsplitting dendritic patterns. Symbols represent the calculated points by the proposed nonlinear phase-field model.

Fig. A1. Landscape of excess chemical potential along the reaction coordinate explored by the electrodeposition reaction, $\mathrm{M}^{n+}+n \mathrm{e}^{-} \rightarrow \mathrm{M}$ at equilibrium (black) and during the reduction reaction (red) under a negative overpotential $\eta<0$. 


\section{List of Tables}

Table 1 Phase-field simulation parameters and their normalized values. 
Fig.1

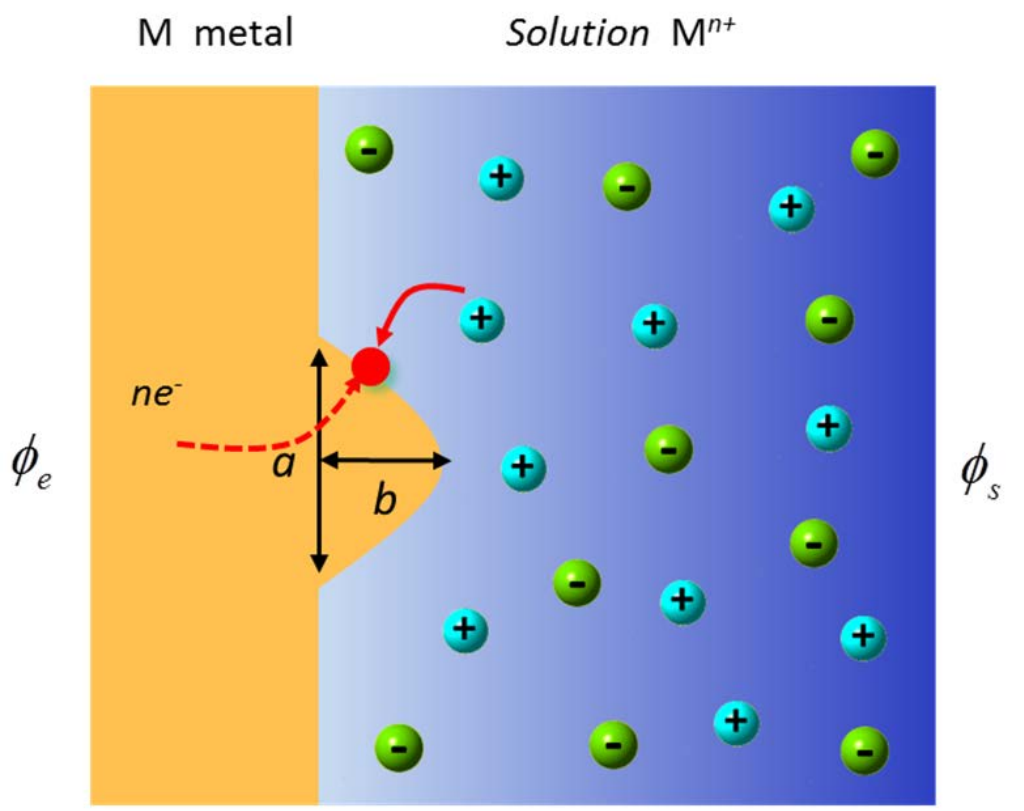


Fig.2

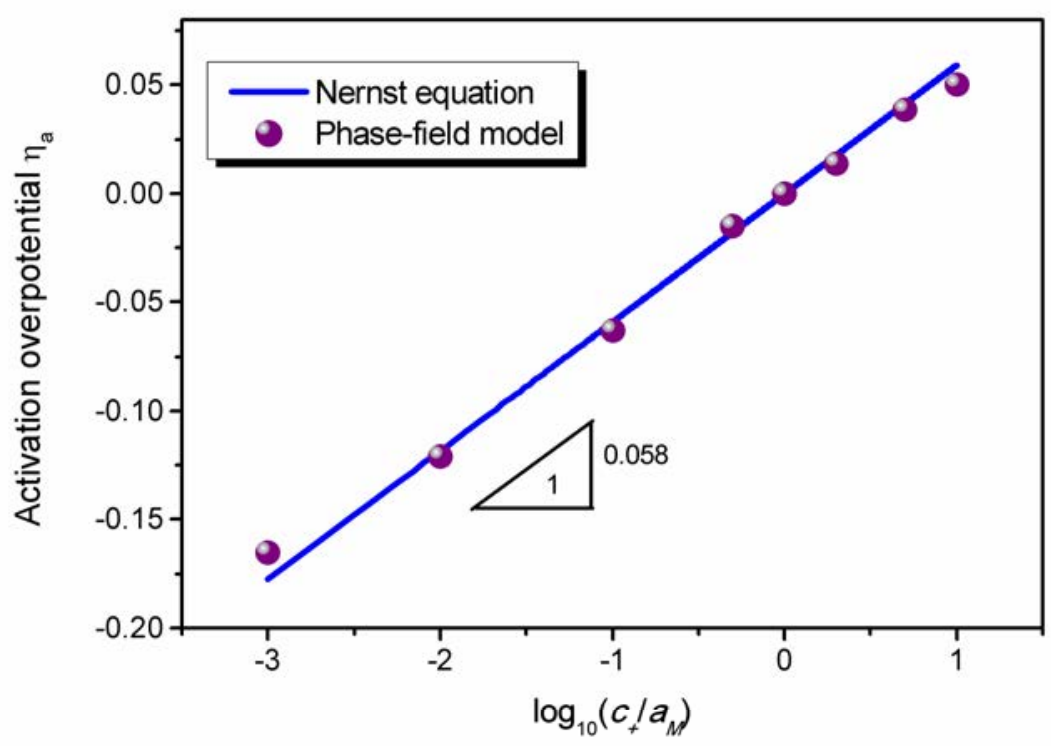


Fig. 3

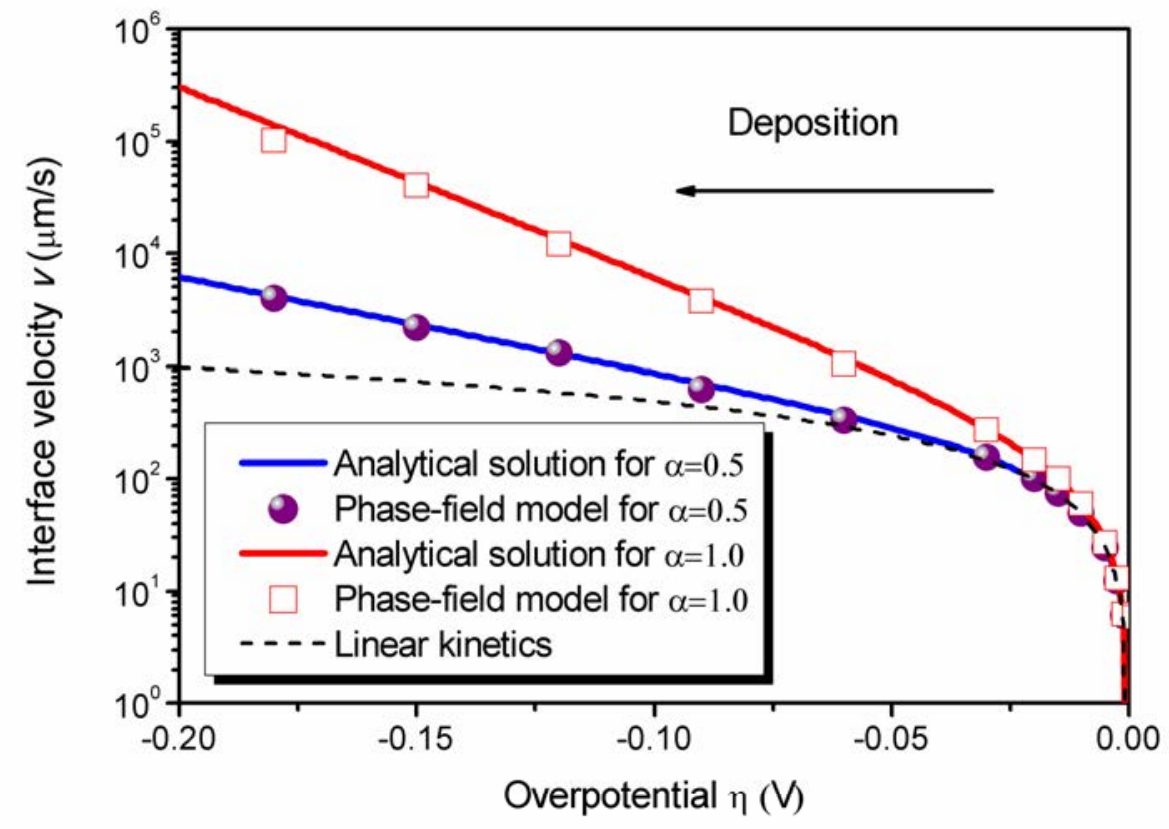


Fig.4

$\mathrm{t}=25 \mathrm{~s}$

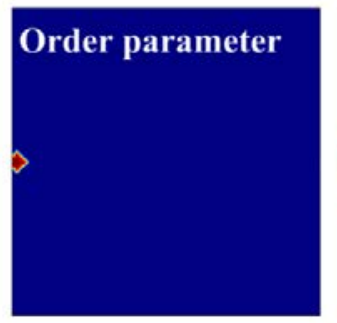

$\mathrm{Li}^{+}$Concentration

D

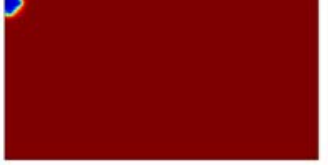

Electric potential

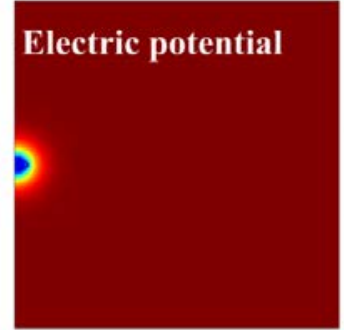

$\mathrm{t}=125 \mathrm{~s}$
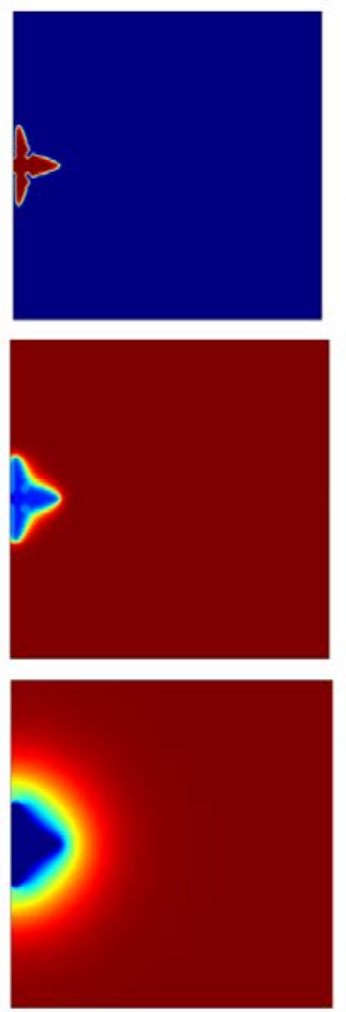

$\mathrm{t}=250 \mathrm{~s}$
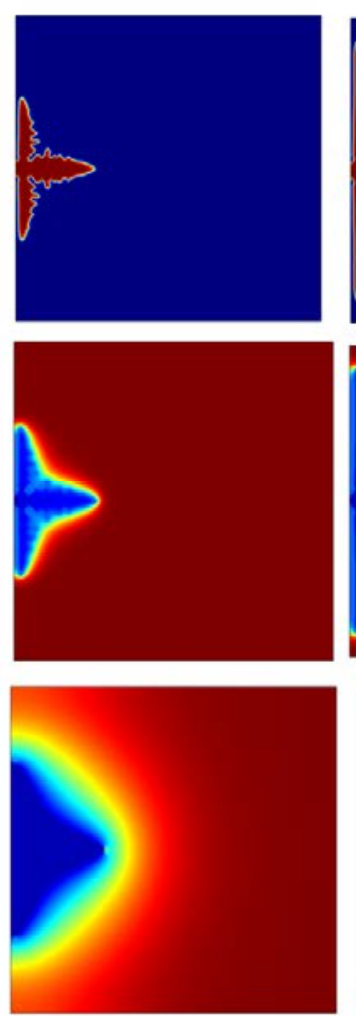

$\mathrm{t}=500 \mathrm{~s}$
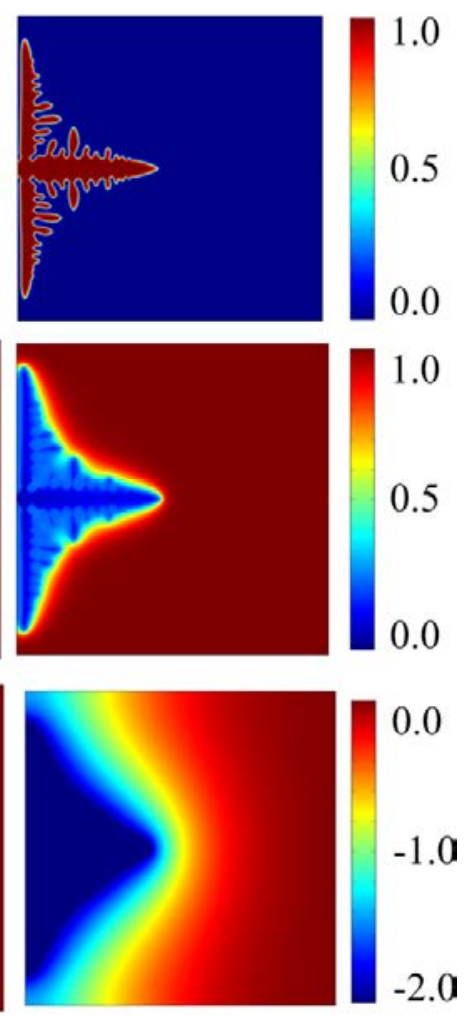
Fig.5

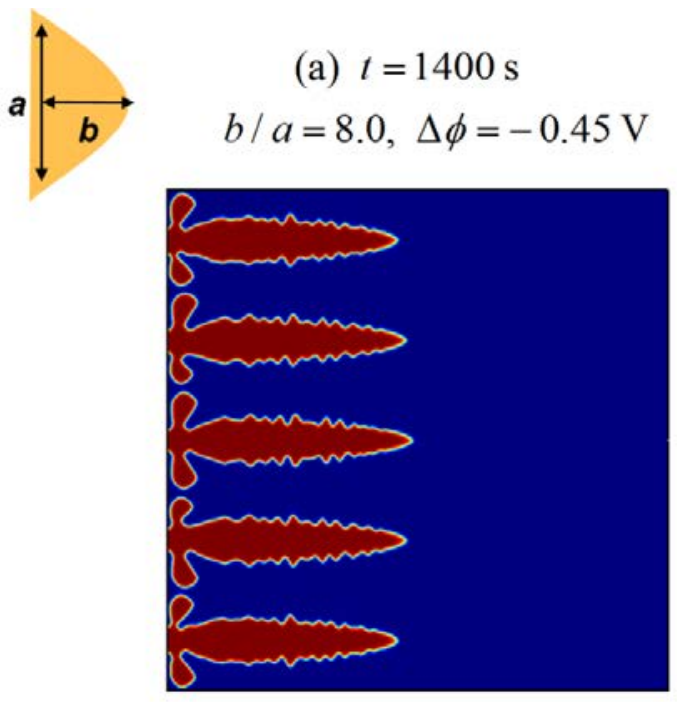

(c) $t=800 \mathrm{~s}$

$b / a=2.0, \Delta \phi=-3.0 \mathrm{~V}$

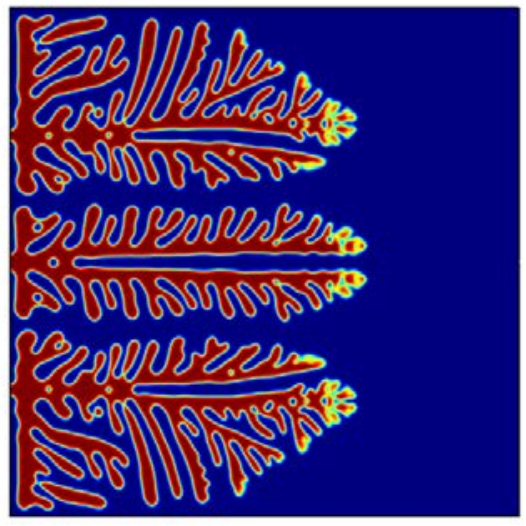

(b) $t=800 \mathrm{~s}$

$b / a=4.0, \Delta \phi=-1.5 \mathrm{~V}$

(d) Experimental observation
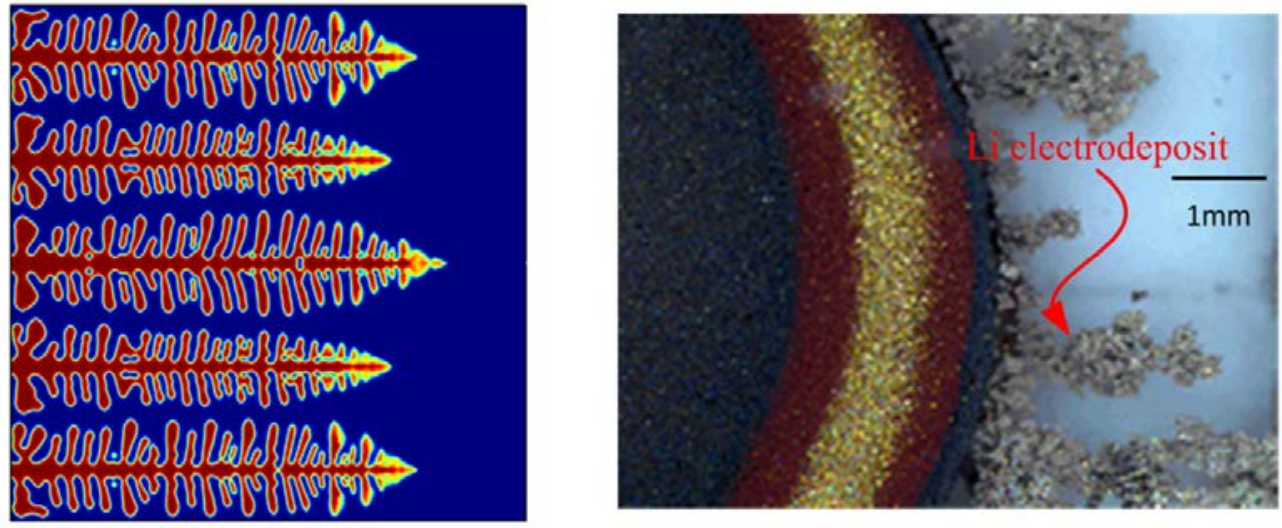
Fig.6

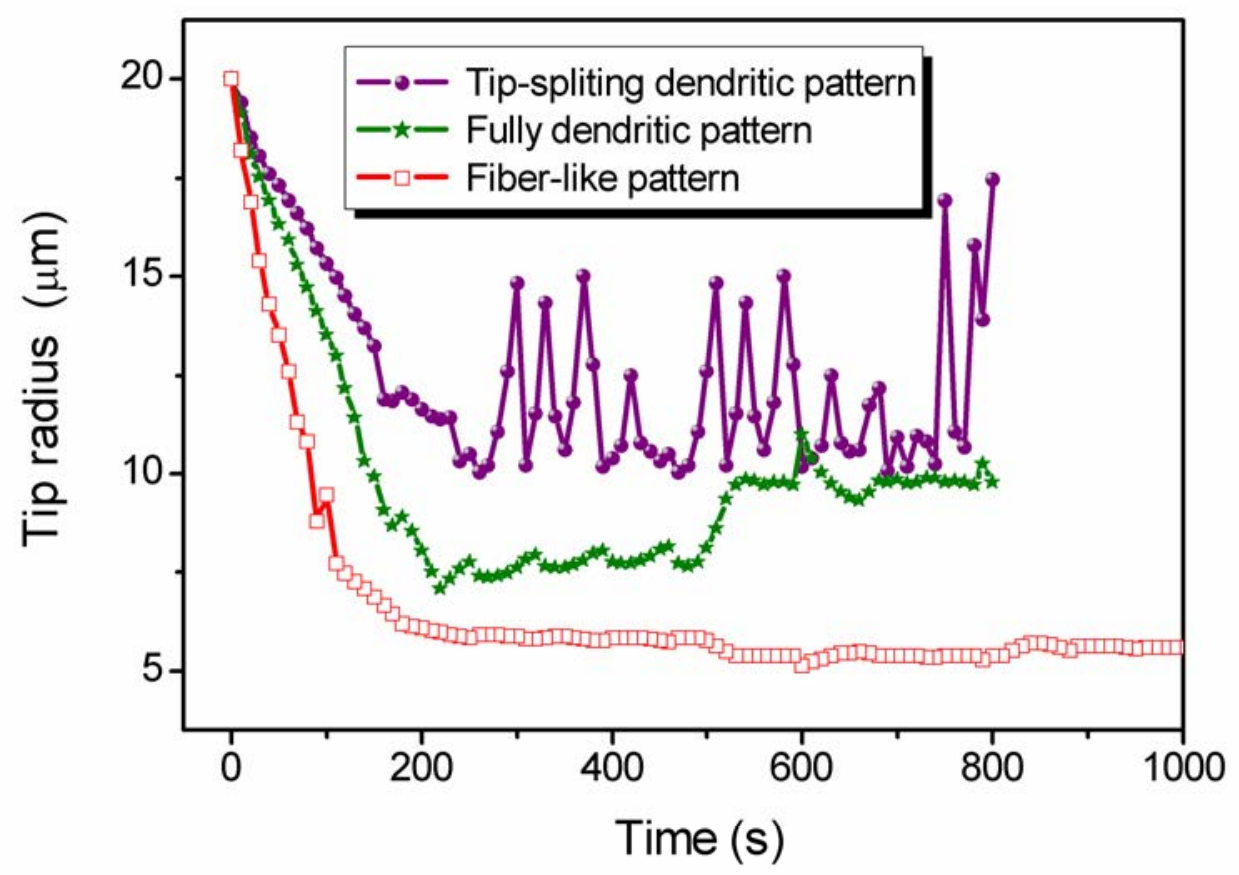


Fig.7

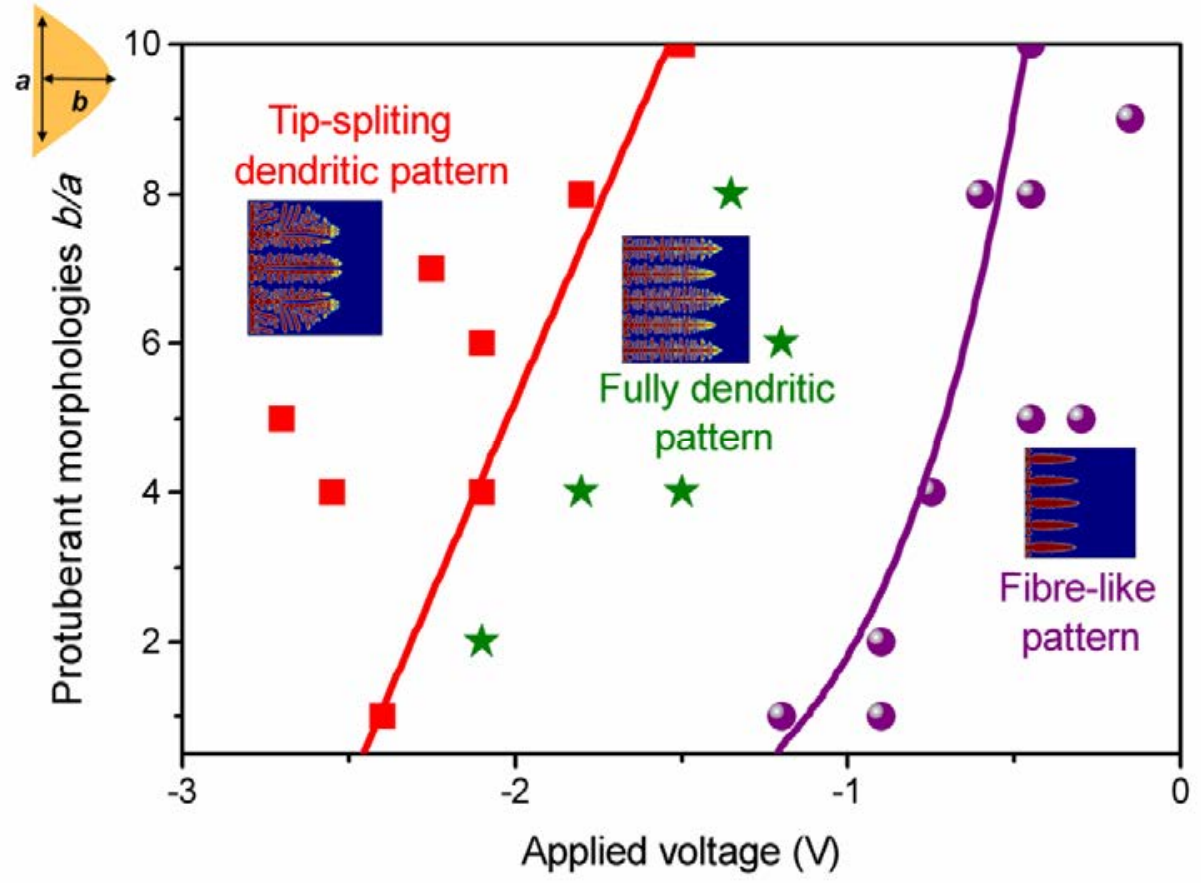


Fig. A1

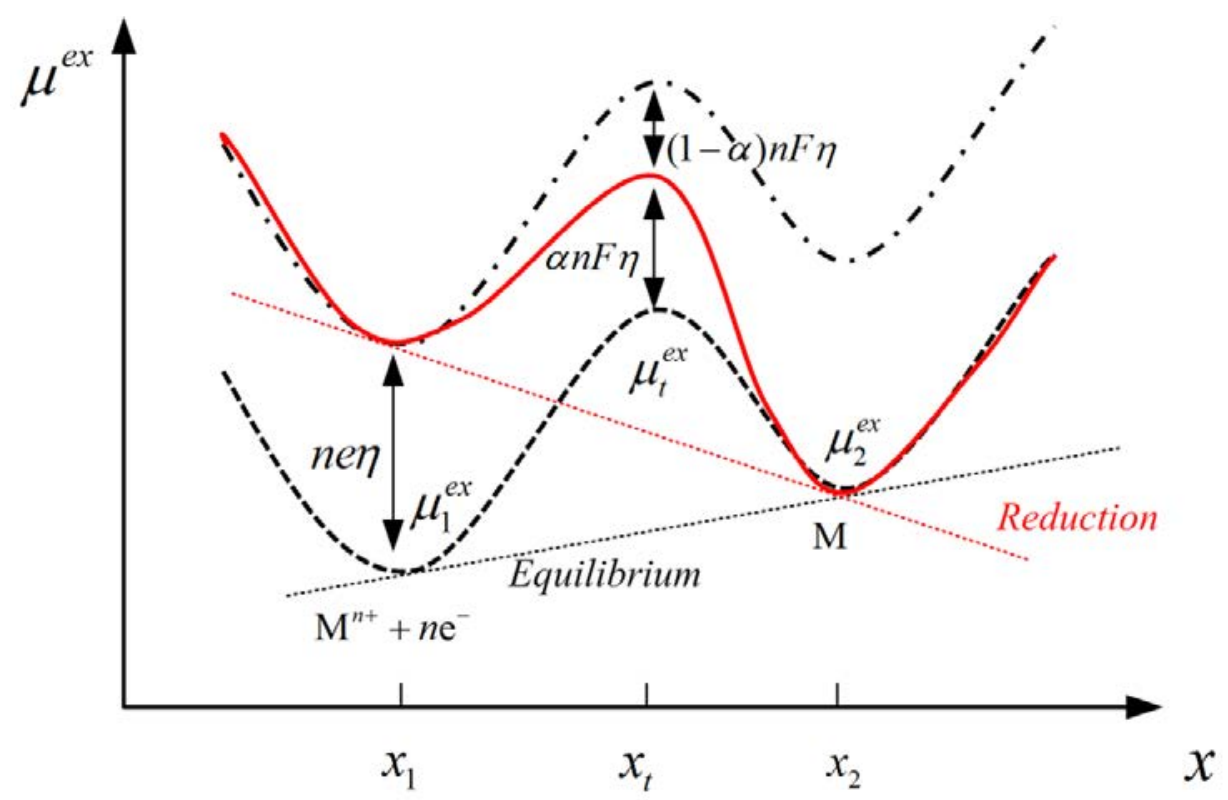


Table 1

\begin{tabular}{|c|c|c|c|c|}
\hline \multirow[b]{2}{*}{ Parameter } & \multicolumn{2}{|c|}{ Real value } & \multicolumn{2}{|c|}{ Normalized value } \\
\hline & Symbol & Value & Symbol & Value \\
\hline Interfacial mobility & $L_{\sigma}$ & $2.5 \times 10^{-6} \mathrm{~m}^{3} /(\mathrm{J} \times \mathrm{s})$ & $\tilde{L}_{\sigma}=L_{\sigma} \times\left(E_{0} \times \Delta t_{0}\right)$ & 2000 \\
\hline Reaction constant. & $L_{\eta}$ & $1.0 / \mathrm{s}$ & $\tilde{L}_{\eta}=L_{\eta} \times \Delta t_{0}$ & 4000 \\
\hline Gradient energy coeff. & $\kappa$ & $5 \times 10^{-5} \mathrm{~J} / \mathrm{m}$ & $\tilde{\kappa}=\kappa /\left(E_{0} \times l_{0}^{2}\right)$ & 0.01 \\
\hline Interfacial energy & $\gamma$ & $0.6 \mathrm{~J} / \mathrm{m}^{2}$ & $\tilde{\gamma}=\gamma /\left(E_{0} \times l_{0}\right)$ & 0.004 \\
\hline Barrier height & $W$ & $3.75 \times 10^{5} \mathrm{~J} / \mathrm{m}^{3}$ & $\tilde{W}=W / E_{0}$ & 0.25 \\
\hline System size & $l$ & $500 \mu \mathrm{m}$ & $\tilde{l}=l / l_{0}$ & 5.0 \\
\hline Time step & $\Delta t$ & $0.2 \mathrm{~s}$ & $\Delta \tilde{t}=\Delta t / \Delta t_{0}$ & $5 \times 10^{-5}$ \\
\hline Diffusion coeff. in electrode & $D^{e}$ & $7.5 \times 10^{-13} \mathrm{~m}^{2} / \mathrm{s}$ & $\tilde{D}^{e}=D^{e} /\left(l_{0}^{2} / \Delta t_{0}\right)$ & 0.03 \\
\hline Diffusion coeff. in solution & $D^{s}$ & $7.5 \times 10^{-10} \mathrm{~m}^{2} / \mathrm{s}$ & $\tilde{D}^{s}=D^{s} /\left(l_{0}^{2} / \Delta t_{0}\right)$ & 30 \\
\hline Conductivity in electrode & $\sigma^{e}$ & $1.0 \times 10^{7} \mathrm{~S} / \mathrm{m}$ & $\tilde{\sigma}^{e}=\sigma^{e} /\left(\frac{l_{0}^{2}}{\Delta t_{0}} \cdot \frac{c_{0} F^{2}}{R T}\right)$ & $10^{9}$ \\
\hline Conductivity in solution & $\sigma^{s}$ & $1.0 \mathrm{~S} / \mathrm{m}$ & $\tilde{\sigma}^{s}=\sigma^{s} /\left(\frac{l_{0}^{2}}{\Delta t_{0}} \cdot \frac{c_{0} F^{2}}{R T}\right)$ & 100 \\
\hline
\end{tabular}

